\begin{tabular}{|c|c|c|c|c|}
\hline Fluid Type & $\begin{array}{l}\text { Density } \\
\text { (kg/cu.m) }\end{array}$ & $\begin{array}{l}\text { Dynamic } \\
\text { Viscosity } \\
\left(\mathrm{Pa}^{*} \mathrm{~s}\right)\end{array}$ & $\begin{array}{l}\text { Relative Error } \\
\text { of Density against } \\
\text { human blood }\end{array}$ & $\begin{array}{l}\text { Relative Error } \\
\text { of viscosity against } \\
\text { human blood }\end{array}$ \\
\hline Water & 998 & 0.001002 & $5.85 \%$ & $71.67 \%$ \\
\hline $\begin{array}{l}\text { Bovine Blood } \\
\text { (cow) }\end{array}$ & 1045.9 & 0.003 & $1.33 \%$ & $15.19 \%$ \\
\hline $\begin{array}{l}\text { Glycerol mixture } \\
\text { (22\% wt.) }\end{array}$ & 1052.9 & 0.00124 & $0.70 \%$ & $64.95 \%$ \\
\hline 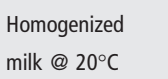 & 1033 & 0.002 & $2.55 \%$ & $43.46 \%$ \\
\hline CMC (1\% wt.) & 1000.4 & 0.003002 & $5.28 \%$ & $15.14 \%$ \\
\hline $\begin{array}{l}\text { Human Blood at } \\
\text { CW }\end{array}$ & 1060 & 0.0035375 & None & None \\
\hline
\end{tabular}

Results We compared the density and dynamic viscosity of different materials in table 1. Different CMC fluid concentrations and the resulting viscosity ranges are compared to blood with increasing shear rate (figure 1). CMC matches the initial viscosity and undergoes shear-thinning like human blood. Distilled water has a lower viscosity than blood that does not change with shear rate. It was found that CMC (1\% wt) is closest to the material properties of human blood flow at the Circle of Willis (CW).

Conclusion CMC fluid is a promising tool in investigating blood flow characteristics in a bench-top model. It can mimic accurate physiologically relevant mechanical properties of blood and has the potential of being used as a go-to tool for medical device testing applications. This fluid will be able to minimize friction between synthetic vessels and endovascular devices and enhance the accuracy of simulated surgery models. Disclosures H. Sodawalla: None. W. Merritt: None. T. Becker: 1; C; NIH STTR Phase I (\#1R41NS097069-01A1).

\section{E-205 CLIP LIGATION OF AN ANTERIOR COMMUNICATING ARTERY ANEURYSM WITH DELAYED RUPTURE AFTER WEB EMBOLIZATION: A CASE REPORT}

N Villelli*, B Bohnstedt. Department of Neurological Surgery, Indiana University, Indianapolis, IN
Introduction The Woven EndoBridge (WEB) aneurysm embolization system has emerged as a new and effective endovascular treatment option for broad necked cerebral aneurysms located at bifurcations. Intra-procedural hemorrhage is a known complication of the device, but we report aneurysmal rupture two days after WEB treatment of an unruptured anterior communicating artery aneurysm. Additionally, instances of surgical clip ligation of aneurysms previously treated with WEB are scarce in the literature. We describe our experience of successful clip ligation of an aneurysm previously treated with WEB.

Methods A 62 year-old female underwent an uneventful WEB embolization of an unruptured anterior communicating artery aneurysm, which measured $3 \mathrm{~mm} \times 4 \mathrm{~mm}$ in maximal diameter. The WEB device used measured $4 \mathrm{~mm} \times 3 \mathrm{~mm}$. Final angiogram demonstrated decreased opacification of the aneurysm dome. The patient was discharged home on post-procedure day 1 on Aspirin $81 \mathrm{mg}$ daily, but returned the following evening with worsening headache. Head CT revealed an acute subarachnoid hemorrhage (SAH) centered on the treated aneurysm. She remained neurologically intact. Formal angiogram after rupture exhibited increased intrasaccular opacification of the aneurysm.

Results The patient was monitored in the ICU and placed on our institution's SAH management protocol including levetiracetam, nimodipine, atorvastatin, intravenous fluids and early DVT prophylaxis. She developed one episode of symptomatic vasospasm requiring intra-arterial milrinone therapy on posthemorrhage day 6. Follow-up angiogram demonstrated continued filling of aneurysm through the WEB device. The device itself appeared to still be under compression, but due to the delayed hemorrhage, further treatment was thought to be appropriate. The patient was subsequently taken to the operating room on post-hemorrhage day 21 for clip ligation of the aneurysm. A standard pterional approach was used. Clip placement was performed without issue and there were no significant challenges faced due to the presence of the WEB device. Post-operative angiogram confirmed aneurysm occlusion. The patient was discharged home on post-operative day 4 , and remained neurologically intact.

Conclusion To our knowledge, this is the first reported case of a delayed aneurysmal subarachnoid hemorrhage after WEB embolization. Additionally, clip ligation of an aneurysm previously treated with WEB can be done safely, and in our patient, did not result in a more challenging operation.

Disclosures N. Villelli: None. B. Bohnstedt: None.

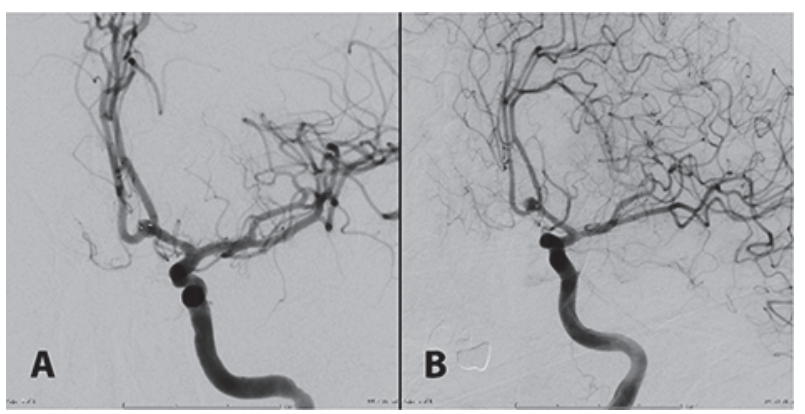

Abstract E-205 Figure 1 A) Initial post-embolization angiogram after WEB deployment demonstrating appropriate placement of the device 